\title{
Development of Chiral Pincer Palladium Complexes Bearing a Pyrroloimidazolone Unit. Catalytic Use for Asymmetric Michael Addition
}

\author{
Kazuhiro Takenaka and Yasuhiro Uozumi* \\ Institute for Molecular Science (IMS), Myodaiji, Okazaki 444-8787, Japan \\ uo@ims.ac.jp
}

SUPPORTING INFORMATION

General: All manipulations were carried out under a nitrogen atmosphere. Nitrogen gas was dried by passage through $\mathrm{P}_{2} \mathrm{O}_{5}$. NMR spectra were recorded on a JEOL JNM-AL400 spectrometer (400 MHz for ${ }^{1} \mathrm{H}, 100 \mathrm{MHz}$ for ${ }^{13} \mathrm{C}, 162 \mathrm{MHz}$ for ${ }^{31} \mathrm{P}$ ), JEOL JNM-AL500 spectrometer $\left(500 \mathrm{MHz}\right.$ for ${ }^{1} \mathrm{H}, 125 \mathrm{MHz}$ for ${ }^{13} \mathrm{C}$ ), or JEOL JNM-LA500 spectrometer $\left(500 \mathrm{MHz}\right.$ for ${ }^{1} \mathrm{H}, 125 \mathrm{MHz}$ for $\left.{ }^{13} \mathrm{C}\right)$. Chemical shifts are reported in $\delta \mathrm{ppm}$ referenced to an internal tetramethylsilane standard for ${ }^{1} \mathrm{H}$ NMR. Chemical shifts of ${ }^{13} \mathrm{C}$ NMR are given relative to $\mathrm{CDCl}_{3}$ as an internal standard $\left(d\right.$ 77.0). The ${ }^{31} \mathrm{P}$ NMR data are reported relative to external $85 \% \mathrm{H}_{3} \mathrm{PO}_{4}$. ${ }^{1} \mathrm{H},{ }^{13} \mathrm{C}$, and ${ }^{31} \mathrm{P}$ NMR spectra were recorded in $\mathrm{CDCl}_{3}$ at $25{ }^{\circ} \mathrm{C}$ unless otherwise noted. FAB mass spectra were recorded on a JEOL JMS-777V spectrometer; 3-nitrobenzyl alcohol was used as the matrix. Melting points were determined using a Yanaco micro melting point apparatus MP-J3 and are uncorrected. Optical rotations were measured on a JASCO P-1020 polarimeter. GC analysis was carried out on a Hewlett Packard 4890 system with a chiral stationary phase column, Cyclodex CB. Commercially available reagents were used without any purification. 5-tert-Butyl-1,3-diformyl-2-hydroxybenzene ${ }^{1}$ and $\mathrm{Pd}_{2}\left(\mathrm{dba}_{3} \cdot \mathrm{CHCl}_{3}{ }^{2}\right.$ were prepared by the literature methods. Anilides 6 and 7 were prepared by the methods reported by our laboratory. ${ }^{3} \quad$ Isopropyl 2-cyanopropionate (9a) and diisopropylmethyl 2cyanopropionate $(\mathbf{9 c})$ were prepared according to procedures in the literature. ${ }^{4}$

4-tert-Butyl-2,6-diformylphenyl trifluoromethanesulfonate. To a solution of 5-tert-butyl-1,3-diformyl-2hydroxybenzene $^{1}(1.72 \mathrm{~g}, 8.34 \mathrm{mmol})$ and pyridine $(1.20$ $\mathrm{mL}, 14.8 \mathrm{mmol})$ in $50 \mathrm{~mL}$ of $\mathrm{CH}_{2} \mathrm{Cl}_{2}$ was added trifluoromethanesulfonic anhydride $(1.80 \mathrm{ml}, 10.7 \mathrm{mmol})$ dropwise at $0{ }^{\circ} \mathrm{C}$ over $10 \mathrm{~min}$. The reaction mixture was warmed to room temperature and stirred for $30 \mathrm{~min}$. The solution was cooled to $0{ }^{\circ} \mathrm{C}$ and quenched with $40 \mathrm{~mL}$ of $1.5 \mathrm{M} \mathrm{HCl}$. After separation, the aqueous phase was extracted twice with $\mathrm{CH}_{2} \mathrm{Cl}_{2}(30 \mathrm{~mL})$ and the combined extract was dried over $\mathrm{Na}_{2} \mathrm{SO}_{4}$. The organic phase was passed through a short plug of silica gel, which was then washed with $\mathrm{CH}_{2} \mathrm{Cl}_{2}(100 \mathrm{~mL})$. The filtrates were concentrated under reduced pressure and the crude product was recrystallized from hexane to give the title compound $(2.63 \mathrm{~g}, 93 \%)$ as a white crystalline solid. Mp: $69-70{ }^{\circ} \mathrm{C}$. MS (EI): $m / z 338\left([\mathrm{M}]^{+}\right) .{ }^{1} \mathrm{H} \mathrm{NMR}\left(\mathrm{CDCl}_{3}, 500 \mathrm{MHz}\right): \delta$ 1.35 (s, 9H, $\mathrm{CH}_{3}$ ), 8.22 (s, 2H, $\left.\mathrm{ArH}\right), 10.24$ (s, 2H, CHO); ${ }^{13} \mathrm{C}\left\{{ }^{1} \mathrm{H}\right\}$ NMR $\left(\mathrm{CDCl}_{3}, 125 \mathrm{MHz}\right): \delta 31.1\left(\mathrm{C}\left(\mathrm{CH}_{3}\right)_{3}\right), 35.5$ $\left(\mathrm{C}\left(\mathrm{CH}_{3}\right)_{3}\right), 118.4\left(\mathrm{q},{ }^{1} J_{\mathrm{CF}}=320 \mathrm{~Hz}, \mathrm{O}_{3} \mathrm{SCF}_{3}\right), 129.2(\mathrm{ArC}$ attached to $\mathrm{CHO}$ ), 132.4 ( $\mathrm{ArC}$ ), 147.0 (ArC attached to $t$ $\mathrm{Bu}$ ), 153.2 (ArC attached to OTf), 185.3 ( $\mathrm{CHO}$ ). Anal. Calcd for $\mathrm{C}_{13} \mathrm{H}_{13} \mathrm{~F}_{3} \mathrm{O}_{5} \mathrm{~S}$ : C, 46.15; H, 3.87; S, 9.48. Found: C, 45.89; H, 3.87; S, 9.64.
trans-(4-tert-Butyl-2,6-diformylphenyl)chlorobis(triphenylphosphine)palladium (5). 4-tert-Butyl-2,6diformylphenyl trifluoromethanesulfonate $(2.03 \mathrm{~g}, \quad 6.00$ mmol), $\quad \mathrm{Pd}_{2}(\mathrm{dba})_{3} \cdot \mathrm{CHCl}_{3}{ }^{2}(3.11 \mathrm{~g}, 3.00 \mathrm{mmol})$, and triphenylphosphine $(3.15 \mathrm{~g}, 12.0 \mathrm{mmol})$ were dissolved in $60 \mathrm{~mL}$ of $\mathrm{CH}_{2} \mathrm{Cl}_{2}$, which was stirred at room temperature for $4 \mathrm{~h}$. After removal of the solvent, the residue and $\mathrm{LiCl}$ (2.73 g, $64 \mathrm{mmol})$ were suspended in a mixture of acetone $(50 \mathrm{~mL})$ and water $(10 \mathrm{~mL})$. The reaction mixture was stirred at room temperature for $10 \mathrm{~h}$ and then concentrated under reduced pressure. The residue was dissolved in 100 $\mathrm{mL}$ of $\mathrm{CHCl}_{3}$, which was washed with water $(50 \mathrm{~mL} \times 2)$ and brine $(50 \mathrm{~mL})$, and dried over $\mathrm{Na}_{2} \mathrm{SO}_{4}$. After evaporation of the solvent, the resulting solid was recrystallized from $\mathrm{MeCN}$ to afford 5 (4.71 g, 92\%) as a yellow crystal. Mp: $226-229^{\circ} \mathrm{C}$ (dec.). MS (FAB): $\mathrm{m} / \mathrm{z}$ $819\left([\mathrm{M}-\mathrm{Cl}]^{+}\right) . \quad{ }^{1} \mathrm{H} \mathrm{NMR}\left(\mathrm{CDCl}_{3}, 400 \mathrm{MHz}\right): \delta 1.16(\mathrm{~s}$, $\left.9 \mathrm{H}, \mathrm{CH}_{3}\right), 7.15(\mathrm{~s}, 2 \mathrm{H}, \mathrm{Ar} H), 7.22\left(\mathrm{t}, 12 \mathrm{H},{ }^{3} J_{\mathrm{HH}}=7.6 \mathrm{~Hz}\right.$, $m-\mathrm{Ph} H), 7.30\left(\mathrm{t}, 6 \mathrm{H},{ }^{3} J_{\mathrm{HH}}=7.2 \mathrm{~Hz}, p-\mathrm{Ph} H\right), 7.50(\mathrm{dd}, 12 \mathrm{H}$, $o-\mathrm{Ph} H), 10.40(\mathrm{~s}, 2 \mathrm{H}, \mathrm{CHO}) ;{ }^{13} \mathrm{C}\left\{{ }^{1} \mathrm{H}\right\} \mathrm{NMR}\left(\mathrm{CDCl}_{3}, 100\right.$ $\mathrm{MHz}): \delta 31.3\left(\mathrm{C}\left(\mathrm{CH}_{3}\right)_{3}\right), 34.1\left(\mathrm{C}\left(\mathrm{CH}_{3}\right)_{3}\right), 127.9$ (virtual t, $\left.\right|^{2} J_{\mathrm{CP}}+{ }^{4} J_{\mathrm{CP}} \mid=5.3 \mathrm{~Hz}, o-\mathrm{PhC}$ ), 129.7 (virtual t, $\left|{ }^{1} J_{\mathrm{CP}}+{ }^{3} J_{\mathrm{CP}}\right|=$ $23.9 \mathrm{~Hz}$, ipso-PhC), $129.9(p-\mathrm{PhC}), 132.4(\mathrm{ArC}), 134.1$ (virtual t, $\left.\left.\right|^{3} J_{\mathrm{CP}}+{ }^{5} J_{\mathrm{CP}}=6.3 \mathrm{~Hz}, m-\mathrm{Ph} C\right), 140.9$ (ArC 
attached to $\mathrm{CHO}$ ), $146.7(\mathrm{ArC}$ attached to $t-\mathrm{Bu}), 172.4(\mathrm{t}$, ${ }^{2} J_{\mathrm{CP}}=6.2 \mathrm{~Hz}, \mathrm{ArC}$ attached to $\left.\mathrm{Pd}\right), 194.7(C \mathrm{HO}) ;{ }^{31} \mathrm{P}\left\{{ }^{1} \mathrm{H}\right\}$ NMR $\left(\mathrm{CDCl}_{3}, 162 \mathrm{MHz}\right): \delta 22.5$ (s). Anal. Calcd for $\mathrm{C}_{48} \mathrm{H}_{43} \mathrm{ClO}_{2} \mathrm{P}_{2} \mathrm{Pd} \cdot \mathrm{CH}_{3} \mathrm{CN}$ : C, 66.97; H, 5.17; N, 1.56 . Found: C, 67.04; H, 5.17; N, 1.53.

[4-tert-Butyl-2,6-bis $\{(3 R, 7 \mathrm{a} S)-2-p h e n y l h e x a h y d r o-$ $1 H$-pyrrolo[1,2-c]imidazole-1-on-3-yl\}phenyl]chloro-

palladium (1-Cl). The palladium complex $\mathbf{5}(1.71 \mathrm{~g}, 2.00$ $\mathrm{mmol}$ ) and the anilide $\mathbf{6}^{3}$ (3.80 g, $\left.20 \mathrm{mmol}\right)$ were suspended in $60 \mathrm{~mL}$ of MeCN. The suspension was refluxed under an $\mathrm{O}_{2}$ atmosphere for $94 \mathrm{~h}$ (until it turned to a clear solution) and then allowed to cool to room temperature. After removal of the solvent, the crude product was treated with $\mathrm{MeOH}$ and ether. The resulting yellow powder was filtered, washed with ether, and dried in vacuo to give 1-Cl (1.33 g, 98\%). Mp: $263-265{ }^{\circ} \mathrm{C}$ (dec.). $\quad$ MS (FAB): $\mathrm{m} / \mathrm{z}$ $639\left([\mathrm{M}-\mathrm{Cl}]^{+}\right) . \quad[\alpha]_{\mathrm{D}}{ }^{24}-161\left(c 1.0, \mathrm{CH}_{2} \mathrm{Cl}_{2}\right) .{ }^{1} \mathrm{H}$ NMR $\left(\mathrm{CDCl}_{3}, 500 \mathrm{MHz}\right): \delta 0.70\left(\mathrm{~s}, 9 \mathrm{H}, \mathrm{CH}_{3}\right), 1.81(\mathrm{~m}, 2 \mathrm{H}), 2.17$ $(\mathrm{m}, 2 \mathrm{H}), 2.39(\mathrm{~m}, 2 \mathrm{H}), 2.60(\mathrm{~m}, 2 \mathrm{H}), 3.56(\mathrm{~m}, 2 \mathrm{H}), 4.23(\mathrm{~m}$, $2 \mathrm{H}), 5.13\left(\mathrm{dd}, 2 \mathrm{H},{ }^{3} J_{\mathrm{HH}}=9.7 \mathrm{~Hz},{ }^{3} J_{\mathrm{HH}}=1.9 \mathrm{~Hz}\right.$, bridgehead $\mathrm{CH}), 5.87(\mathrm{~s}, 2 \mathrm{H}), 6.15(\mathrm{~s}, 2 \mathrm{H}), 7.20\left(\mathrm{~d}, 4 \mathrm{H},{ }^{3} J_{\mathrm{HH}}=\right.$ $7.6 \mathrm{~Hz}, o-\mathrm{Ph} H), 7.32\left(\mathrm{t}, 2 \mathrm{H},{ }^{3} J_{\mathrm{HH}}=7.5 \mathrm{~Hz}, p-\mathrm{Ph} H\right), 7.41(\mathrm{t}$, $\left.4 \mathrm{H},{ }^{3} J_{\mathrm{HH}}=7.8 \mathrm{~Hz}, m-\mathrm{Ph} H\right) ;{ }^{13} \mathrm{C}\left\{{ }^{1} \mathrm{H}\right\}$ NMR $\left(\mathrm{CDCl}_{3}, 125\right.$ $\mathrm{MHz}): \delta 23.5\left(\mathrm{CH}_{2}\right.$ in pyrroloimidazolone ring), $29.2\left(\mathrm{CH}_{2}\right.$ in pyrroloimidazolone ring), $\left.30.7\left(\mathrm{C}_{(\mathrm{CH}}\right)_{3}\right), \quad 34.2$ $\left(\mathrm{C}\left(\mathrm{CH}_{3}\right)_{3}\right), 63.6\left(\mathrm{~N}-\mathrm{CH}_{2}\right), 71.3$ (bridge-head $\left.\mathrm{CH}\right), 94.4(\mathrm{~N}-$ $C \mathrm{H}-\mathrm{N}), 121.5(p-\mathrm{Ph} C), 126.6(o-\mathrm{Ph} C), 128.0(\mathrm{ArC}), 129.5$ $(m-\mathrm{Ph} C), 134.9$ (ipso-PhC), 144.6 (ArC attached to pyrroloimidazolone ring), 146.7 (ArC attached to $t$-Bu), 150.0 (ArC attached to $\mathrm{Pd}), 170.2(C=\mathrm{O})$. Anal. Calcd for $\mathrm{C}_{34} \mathrm{H}_{37} \mathrm{ClN}_{4} \mathrm{O}_{2} \mathrm{Pd}$ : C, 60.45; H, 5.52; N, 8.29. Found: C, $60.30 ; \mathrm{H}, 5.74 ; \mathrm{N}, 8.03$.

[4-tert-Butyl-2,6-bis $\{(3 R, 6 R, 7 \mathrm{a} S)-6-h y d r o x y-2-$ phenylhexahydro- $1 H$-pyrrolo[1,2-c]imidazole-1-on-3yl\}phenyl]chloropalladium (2-Cl). Following the procedure described for the preparation of $\mathbf{3}$, the reaction of the palladium complex $5(855 \mathrm{mg}, 1.00 \mathrm{mmol})$ with the anilide $7^{3}$ (2.83 g, $\left.9.84 \mathrm{mmol}\right)$ in $40 \mathrm{~mL}$ of MeCN under an $\mathrm{O}_{2}$ atmosphere for $136 \mathrm{~h}$ gave 2 -Cl $(617 \mathrm{mg}, 87 \%)$ as yellow needles after trituration of the crude product with EtOAc and hexane. Mp: 204-205 ${ }^{\circ} \mathrm{C}$ (dec.). MS (FAB): $m / z 671\left([\mathrm{M}-\mathrm{Cl}]^{+}\right) . \quad[\alpha]_{\mathrm{D}}{ }^{22}-180\left(c 1.0, \mathrm{CH}_{2} \mathrm{Cl}_{2}\right) .{ }^{1} \mathrm{H}$ NMR (DMSO-d $6,500 \mathrm{MHz}): \delta 0.70\left(\mathrm{~s}, 9 \mathrm{H}, \mathrm{CH}_{3}\right), 2.59(\mathrm{~m}$, $2 \mathrm{H}), 3.72\left(\mathrm{dd}, 2 \mathrm{H},{ }^{3} J_{\mathrm{HH}}=12.5 \mathrm{~Hz},{ }^{3} J_{\mathrm{HH}}=6.9 \mathrm{~Hz}\right), 4.06(\mathrm{dd}$, $\left.2 \mathrm{H},{ }^{3} J_{\mathrm{HH}}=12.5 \mathrm{~Hz},{ }^{3} J_{\mathrm{HH}}=6.9 \mathrm{~Hz}\right), 4.50(\mathrm{~m}, 2 \mathrm{H}), 5.13(\mathrm{dd}$, $\left.2 \mathrm{H},{ }^{3} J_{\mathrm{HH}}=9.5 \mathrm{~Hz},{ }^{3} J_{\mathrm{HH}}=3.4 \mathrm{~Hz}\right), 5.22\left(\mathrm{~d}, 2 \mathrm{H},{ }^{3} J_{\mathrm{HH}}=8.1\right.$ $\mathrm{Hz}), 6.03(\mathrm{~s}, 2 \mathrm{H}), 6.17(\mathrm{~s}, 2 \mathrm{H}), 7.23\left(\mathrm{~d}, 4 \mathrm{H},{ }^{3} J_{\mathrm{HH}}=8.1 \mathrm{~Hz}\right.$, $o-\mathrm{Ph} H), 7.33\left(\mathrm{t}, 2 \mathrm{H},{ }^{3} J_{\mathrm{HH}}=7.6 \mathrm{~Hz}, p-\mathrm{Ph} H\right), 7.41(\mathrm{t}, 4 \mathrm{H}$, $\left.{ }^{3} J_{\mathrm{HH}}=7.6 \mathrm{~Hz}, m-\mathrm{PhH}\right) ;{ }^{13} \mathrm{C}\left\{{ }^{1} \mathrm{H}\right\}$ NMR (DMSO-d 6.125

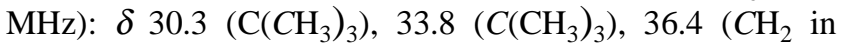
pyrroloimidazolone ring), $67.7\left(\mathrm{~N}-\mathrm{CH}_{2}\right), 68.1(\mathrm{CH}$ in pyrroloimidazolone ring), $69.7(\mathrm{CH}$ in pyrroloimidazolone ring), $93.8(\mathrm{~N}-\mathrm{CH}-\mathrm{N}), 121.3(p-\mathrm{PhC}), 126.1(o-\mathrm{Ph} C), 127.6$ $(\mathrm{ArC}), 129.0 \quad(m-\mathrm{PhC}), 134.3 \quad$ (ipso- $\mathrm{PhC}), 143.3$ ( $\mathrm{ArC}$ attached to pyrroloimidazolone ring), 146.4 (ArC attached to $t$ - $\mathrm{Bu}$ ), 149.7 (ArC attached to $\mathrm{Pd}), 168.9(C=\mathrm{O})$. Anal. Calcd for $\mathrm{C}_{34} \mathrm{H}_{37} \mathrm{ClN}_{4} \mathrm{O}_{4} \mathrm{Pd} \cdot \mathrm{H}_{2} \mathrm{O}$ : C, 56.28; H, 5.42; N, 7.72. Found: C, 56.12; H, 5.37; N, 7.69.

[4-tert-Butyl-2,6-bis $\{(3 R, 7 \mathrm{a} S)-2$-phenylhexahydro-
$1 H$-pyrrolo[1,2-c]imidazole-1-on-3-yl \}phenyl](trifluoromethanesulfonato)palladium (1-OTf). suspension of the chloride complex 1-Cl (338 mg, 0.500 $\mathrm{mmol})$ and silver trifluoromethanesulfonate $(188 \mathrm{mg}, 0.732$ mmol $)$ in a mixture of $\mathrm{CH}_{2} \mathrm{Cl}_{2}(19.8 \mathrm{~mL})$ and water $(0.2$ $\mathrm{mL}$ ) was stirred at room temperature for $16 \mathrm{~h}$. The resulting white precipitate (silver chloride) was removed by filtration through Celite and washed with additional $\mathrm{CH}_{2} \mathrm{Cl}_{2}$. The combined filtrate and washings were evaporated in vacuo to give a yellow powder. The crude product was purified by re-precipitation from $\mathrm{CHCl}_{3}$ and hexane to afford 1-OTf (375 mg, 95\%) as a white powder. Mp: 245-246 ${ }^{\circ} \mathrm{C}$ (dec.). MS (FAB): $\mathrm{m} / \mathrm{z} 639$ ([M OTf $\left.]^{+}\right) . \quad[\alpha]_{\mathrm{D}}{ }^{25}-130 \quad\left(c \quad 0.52, \mathrm{CH}_{2} \mathrm{Cl}_{2}\right) . \quad{ }^{1} \mathrm{H} \quad \mathrm{NMR}$ $\left(\mathrm{CDCl}_{3}, 400 \mathrm{MHz}\right): \delta 0.68\left(\mathrm{~s}, 9 \mathrm{H}, \mathrm{CH}_{3}\right), 1.83(\mathrm{~m}, 2 \mathrm{H}), 2.14$ $(\mathrm{m}, 2 \mathrm{H}), 2.38(\mathrm{~m}, 2 \mathrm{H}), 2.59(\mathrm{~m}, 2 \mathrm{H}), 3.63(\mathrm{~m}, 2 \mathrm{H}), 3.99(\mathrm{~m}$, $2 \mathrm{H}), 4.94\left(\mathrm{dd}, 2 \mathrm{H},{ }^{3} J_{\mathrm{HH}}=9.6 \mathrm{~Hz},{ }^{3} J_{\mathrm{HH}}=2.0 \mathrm{~Hz}\right.$, bridgehead $\mathrm{CH}), 5.74(\mathrm{~s}, 2 \mathrm{H}), 6.10(\mathrm{~s}, 2 \mathrm{H}), 7.12\left(\mathrm{dd}, 4 \mathrm{H},{ }^{3} J_{\mathrm{HH}}=\right.$ $\left.7.2 \mathrm{~Hz},{ }^{3} J_{\mathrm{HH}}=1.2 \mathrm{~Hz}, o-\mathrm{Ph} H\right), 7.34\left(\mathrm{tt}, 2 \mathrm{H},{ }^{3} J_{\mathrm{HH}}=7.6 \mathrm{~Hz}\right.$, $\left.{ }^{3} J_{\mathrm{HH}}=1.2 \mathrm{~Hz}, p-\mathrm{Ph} H\right), 7.40\left(\mathrm{tt}, 4 \mathrm{H},{ }^{3} J_{\mathrm{HH}}=7.4 \mathrm{~Hz},{ }^{3} J_{\mathrm{HH}}=\right.$ $1.2 \mathrm{~Hz}, m-\mathrm{Ph} H) ;{ }^{13} \mathrm{C}\left\{{ }^{1} \mathrm{H}\right\} \mathrm{NMR}\left(\mathrm{CDCl}_{3}, 100 \mathrm{MHz}\right): \delta 23.4$ $\left(\mathrm{CH}_{2}\right.$ in pyrroloimidazolone ring $), 29.2\left(\mathrm{CH}_{2}\right.$ in

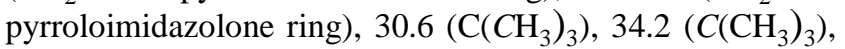
$62.7\left(\mathrm{~N}-\mathrm{CH}_{2}\right), 70.4$ (bridge-head $\left.\mathrm{CH}\right), 93.4(\mathrm{~N}-\mathrm{CH}-\mathrm{N})$, $119.9\left(\mathrm{q},{ }^{1} J_{\mathrm{CF}}=318 \mathrm{~Hz}, \mathrm{O}_{3} \mathrm{SCF}_{3}\right), 122.1(p-\mathrm{PhC}), 127.1(o-$ $\mathrm{PhC}), 128.4(\mathrm{ArC}), 129.5(m-\mathrm{PhC}), 134.1$ (ipso-PhC), 143.8 (ArC attached to $\mathrm{Pd}$ ), 144.6 (ArC attached to pyrroloimidazolone ring), 147.2 (ArC attached to $t$ - $\mathrm{Bu}$ ), $169.6(C=\mathrm{O})$. Anal. Calcd for $\mathrm{C}_{35} \mathrm{H}_{37} \mathrm{~F}_{3} \mathrm{~N}_{4} \mathrm{O}_{5} \mathrm{PdS}$ : C, 53.27; H, 4.73; N, 7.10. Found: C, 53.23; H, 4.72; N, 6.99 .

[4-tert-Butyl-2,6-bis $\{(3 R, 6 R, 7 \mathrm{a} S)-6-h y d r o x y-2-$ phenylhexahydro- $1 H$-pyrrolo[1,2-c]imidazole-1-on-3yl\}phenyl](trifluoromethanesulfonato)palladium OTf). Following the procedure described for the preparation of 1-OTf, reaction of the chloride complex 2-

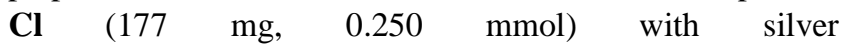
trifluoromethanesulfonate $(81.2 \mathrm{mg}, 0.316 \mathrm{mmol})$ in a mixture of $\mathrm{CH}_{2} \mathrm{Cl}_{2}(19.8 \mathrm{~mL})$ and water $(0.2 \mathrm{~mL})$ at room temperature for $15 \mathrm{~h}$ gave 2-OTf (198 $\mathrm{mg}, 94 \%$ ) as an offwhite powder after trituration of the crude product with EtOAc and ether. Mp: $197-198{ }^{\circ} \mathrm{C}$ (dec.). MS (FAB): $m / z 671\left([\mathrm{M}-\mathrm{OTf}]^{+}\right) . \quad[\alpha]_{\mathrm{D}}{ }^{25}-178(\mathrm{c} 0.52, \mathrm{MeOH}) .{ }^{1} \mathrm{H}$ NMR $\left(\mathrm{CD}_{3} \mathrm{OD}, 400 \mathrm{MHz}\right): \delta 0.71\left(\mathrm{~s}, 9 \mathrm{H}, \mathrm{CH}_{3}\right), 2.35(\mathrm{~m}$, $2 \mathrm{H}), 2.58(\mathrm{~m}, 2 \mathrm{H}), 3.87(\mathrm{~m}, 4 \mathrm{H}), 4.72(\mathrm{~m}, 2 \mathrm{H}), 4.80(\mathrm{dd}$, $\left.2 \mathrm{H},{ }^{3} J_{\mathrm{HH}}=8.8 \mathrm{~Hz},{ }^{3} J_{\mathrm{HH}}=7.2 \mathrm{~Hz}\right), 6.23(\mathrm{~s}, 2 \mathrm{H}), 6.31(\mathrm{~s}$, $2 \mathrm{H}), 7.22\left(\mathrm{dd}, 4 \mathrm{H},{ }^{3} J_{\mathrm{HH}}=6.8 \mathrm{~Hz},{ }^{3} J_{\mathrm{HH}}=1.6 \mathrm{~Hz}, o-\mathrm{Ph} H\right)$, $7.34\left(\mathrm{tt}, 2 \mathrm{H},{ }^{3} J_{\mathrm{HH}}=7.6 \mathrm{~Hz},{ }^{3} J_{\mathrm{HH}}=1.2 \mathrm{~Hz}, p-\mathrm{Ph} H\right), 7.41(\mathrm{tt}$, $\left.4 \mathrm{H},{ }^{3} J_{\mathrm{HH}}=7.6 \mathrm{~Hz},{ }^{3} J_{\mathrm{HH}}=1.2 \mathrm{~Hz}, m-\mathrm{Ph} H\right) ;{ }^{13} \mathrm{C}\left\{{ }^{1} \mathrm{H}\right\} \mathrm{NMR}$ $\left(\mathrm{CD}_{3} \mathrm{OD}, 100 \mathrm{MHz}\right): \delta 31.2\left(\mathrm{C}\left(\mathrm{CH}_{3}\right)_{3}\right), 35.2\left(\mathrm{C}\left(\mathrm{CH}_{3}\right)_{3}\right)$, $37.2\left(\mathrm{CH}_{2}\right.$ in pyrroloimidazolone ring), $68.9\left(\mathrm{~N}-\mathrm{CH}_{2}\right), 70.6$ $(\mathrm{CH}$ in pyrroloimidazolone ring $), \quad 70.9 \quad(\mathrm{CH}$ in pyrroloimidazolone ring), $94.5(\mathrm{~N}-\mathrm{CH}-\mathrm{N}), 121.7$ (q, ${ }^{1} J_{\mathrm{CF}}=$ $\left.318 \mathrm{~Hz}, \mathrm{O}_{3} \mathrm{SCF}_{3}\right), 123.6(p-\mathrm{PhC}), 128.0(o-\mathrm{Ph} C), 129.3$ $(\mathrm{ArC}), 130.4 \quad(m-\mathrm{Ph} C), 135.6$ (ipso-PhC), 145.3 ( $\mathrm{ArC}$ attached to pyrroloimidazolone ring), 146.9 (ArC attached to $\mathrm{Pd}$ ), 148.8 ( $\mathrm{ArC}$ attached to $t$ - $\mathrm{Bu}), 170.5(C=\mathrm{O})$. Anal. Calcd for $\mathrm{C}_{35} \mathrm{H}_{37} \mathrm{~F}_{3} \mathrm{~N}_{4} \mathrm{O}_{7} \mathrm{PdS} \cdot \mathrm{H}_{2} \mathrm{O}$ : C, 50.09; H, 4.68; N, 6.68. Found: C, 50.23; H, 4.75; N, 6.48. 
[4-tert-Butyl-2,6-bis $\{(3 R, 6 R, 7 \mathrm{a} S)-6$-methoxy-2phenylhexahydro-1 $H$-pyrrolo[1,2-c]imidazole-1-on-3yl\}phenyl](trifluoromethanesulfonato)palladium

OTf). To a solution of the chloride complex 2-Cl (70.8 $\mathrm{mg}, 0.100 \mathrm{mmol}$ ) in $10 \mathrm{~mL}$ of THF cooled to $0{ }^{\circ} \mathrm{C}$ was added potassium tert-butoxide (45.0 $\mathrm{mg}, 0.401 \mathrm{mmol}$ ), which was warmed to room temperature and stirred for 1.5 h. The solution was again cooled to $0{ }^{\circ} \mathrm{C}$ and quenched with methyl iodide $(0.15 \mathrm{~mL}, 2.4 \mathrm{mmol})$. After stirring at room temperature for $3 \mathrm{~h}$, the solvent and an excess of methyl iodide were removed by evaporation. The resulting residue was dissolved in $\mathrm{CH}_{2} \mathrm{Cl}_{2}(20 \mathrm{~mL})$, which was washed with water $(20 \mathrm{~mL})$. After separation, the aqueous phase was extracted twice with $\mathrm{CH}_{2} \mathrm{Cl}_{2}(20 \mathrm{~mL})$ and the combined extract was dried over $\mathrm{Na}_{2} \mathrm{SO}_{4}$ and $\mathrm{Na}_{2} \mathrm{~S}_{2} \mathrm{O}_{3}$. The solution was evaporated to give a yellow sludge. The residue and silver trifluoromethanesulfonate (40.9 $\mathrm{mg}, 0.159 \mathrm{mmol}$ ) were suspended in a mixture of $\mathrm{CH}_{2} \mathrm{Cl}_{2}(9.9 \mathrm{~mL})$ and water $(0.1 \mathrm{~mL})$, which was stirred at room temperature for $12 \mathrm{~h}$. The resulting yellow precipitate (silver iodide) was removed by filtration through Celite and washed with additional $\mathrm{CH}_{2} \mathrm{Cl}_{2}$. After evaporation of the solvent, the crude product was chromatographed on silica gel (eluent: $\mathrm{CH}_{2} \mathrm{Cl}_{2} / \mathrm{MeOH}=$ 200/1) and then triturated with hexane to afford 3-OTf (64.8 $\mathrm{mg}, 76 \%$ for 2 steps) as a yellow powder. Mp: 176-178 ${ }^{\circ} \mathrm{C}$ (dec.). MS (FAB): $m / z 699$ ([M - OTf $]^{+}$). $[\alpha]_{\mathrm{D}}{ }^{25}-132\left(c 0.53, \mathrm{CH}_{2} \mathrm{Cl}_{2}\right) .{ }^{1} \mathrm{H} \mathrm{NMR}\left(\mathrm{CDCl}_{3}, 500\right.$ $\mathrm{MHz}): \delta 0.77\left(\mathrm{~s}, 9 \mathrm{H}, \mathrm{C}\left(\mathrm{CH}_{3}\right)_{3}\right), 2.59(\mathrm{~m}, 2 \mathrm{H}), 2.89(\mathrm{~m}, 2 \mathrm{H})$, $3.42\left(\mathrm{~s}, 6 \mathrm{H}, \mathrm{OCH}_{3}\right), 3.57\left(\mathrm{dd}, 2 \mathrm{H},{ }^{3} J_{\mathrm{HH}}=13.5 \mathrm{~Hz},{ }^{3} J_{\mathrm{HH}}=\right.$ $7.5 \mathrm{~Hz}), 4.16(\mathrm{~m}, 2 \mathrm{H}), 4.23\left(\mathrm{~d}, 2 \mathrm{H},{ }^{3} J_{\mathrm{HH}}=14.0 \mathrm{~Hz}\right), 4.88(\mathrm{~d}$, $\left.2 \mathrm{H},{ }^{3} J_{\mathrm{HH}}=8.5 \mathrm{~Hz}\right), 6.30(\mathrm{~s}, 2 \mathrm{H}), 6.43(\mathrm{br} \mathrm{s}, 2 \mathrm{H}), 7.31(\mathrm{t}$, $\left.2 \mathrm{H},{ }^{3} J_{\mathrm{HH}}=7.5 \mathrm{~Hz}, p-\mathrm{Ph} H\right), 7.45\left(\mathrm{t}, 4 \mathrm{H},{ }^{3} J_{\mathrm{HH}}=8.0 \mathrm{~Hz}, m-\right.$ $\mathrm{Ph} H), 7.50\left(\mathrm{~d}, 4 \mathrm{H},{ }^{3} J_{\mathrm{HH}}=8.0 \mathrm{~Hz}, o-\mathrm{Ph} H\right) ;{ }^{13} \mathrm{C}\left\{{ }^{1} \mathrm{H}\right\} \mathrm{NMR}$ $\left(\mathrm{CDCl}_{3}, 125 \mathrm{MHz}\right): \delta 30.8\left(\mathrm{C}\left(\mathrm{CH}_{3}\right)_{3}\right), 33.1\left(\mathrm{CH}_{2}\right.$ in

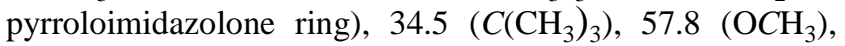
$65.0\left(\mathrm{~N}-\mathrm{CH}_{2}\right), 70.6(\mathrm{CH}$ in pyrroloimidazolone ring), 78.6 $(\mathrm{CH}$ in pyrroloimidazolone ring), $90.0(\mathrm{~N}-\mathrm{CH}-\mathrm{N}), 120.5(\mathrm{q}$, $\left.{ }^{1} J_{\mathrm{CF}}=319 \mathrm{~Hz}, \mathrm{O}_{3} \mathrm{SCF}_{3}\right), 121.0(p-\mathrm{PhC}), 123.5(\mathrm{ArC})$, $127.1(o-\mathrm{Ph} C), 129.4(m-\mathrm{PhC}), 135.3$ (ipso- $\mathrm{PhC}), 141.9$ (ArC attached to pyrroloimidazolone ring), 148.8 ( $\mathrm{ArC}$ attached to $t$-Bu), $168.3(C=\mathrm{O})$; $A r C$ attached to $\mathrm{Pd}$ was not observed. Anal. Calcd for $\mathrm{C}_{37} \mathrm{H}_{41} \mathrm{~F}_{3} \mathrm{~N}_{4} \mathrm{O}_{7} \mathrm{PdS}$ : C, 52.33; $\mathrm{H}$, $4.87 ; \mathrm{N}, 6.60$. Found: C, 52.01; H, 5.27; N, 6.31.

[4-tert-Butyl-2,6-bis $\{(3 R, 6 R, 7 \mathrm{aS})-6$-tert-butyldimethylsilyloxy-2-phenylhexahydro- $1 H$-pyrrolo[1,2c] imidazole-1-on-3-yl\}phenyl](trifluoromethanesulfonato)palladium (4-OTf). To a solution of the complex 2-Cl (283 mg, $0.399 \mathrm{mmol})$ and imidazole (223 $\mathrm{mg}, 3.28 \mathrm{mmol}$ ) in $4.0 \mathrm{~mL}$ of $\mathrm{DMF}$ was added tertbutyldimethylsilyl chloride (306 $\mathrm{mg}, 2.03 \mathrm{mmol}$ ) at $0{ }^{\circ} \mathrm{C}$. The reaction mixture was stirred at room temperature for 1 $\mathrm{h}$ and then quenched with $40 \mathrm{~mL}$ of saturated aqueous $\mathrm{NaHCO}_{3}$. The reaction mixture was extracted three times with $\mathrm{CHCl}_{3}(40 \mathrm{~mL})$ and the combined extract was dried over $\mathrm{Na}_{2} \mathrm{SO}_{4}$. After removal of the solvent, the residue was chromatographed on silica gel (eluent: $\mathrm{CH}_{2} \mathrm{Cl}_{2} / \mathrm{MeOH}$ $=200 / 1)$, followed by recrystallization from a EtOAc-hexane mixture to give the corresponding chloride complex (321 mg, 86\%) as a pale-yellow crystalline solid. Mp: $254-256{ }^{\circ} \mathrm{C}$ (dec.). $\mathrm{MS}$ (FAB): $m / z 899\left([\mathrm{M}-\mathrm{Cl}]^{+}\right)$. $\left.[\alpha]_{\mathrm{D}}{ }^{22}-105(c) 1.0, \mathrm{CH}_{2} \mathrm{Cl}_{2}\right) .{ }^{1} \mathrm{H}$ NMR $\left(\mathrm{CDCl}_{3}, 400\right.$ $\mathrm{MHz}): \delta 0.10\left(\mathrm{~s}, 6 \mathrm{H}, \mathrm{SiCH}_{3}\right), 0.11\left(\mathrm{~s}, 6 \mathrm{H}, \mathrm{SiCH}_{3}\right), 0.68(\mathrm{~s}$, $\left.9 \mathrm{H}, \mathrm{C}\left(\mathrm{CH}_{3}\right)_{3}\right), 0.90\left(\mathrm{~s}, 18 \mathrm{H}, \mathrm{SiC}\left(\mathrm{CH}_{3}\right)_{3}\right), 2.53\left(\mathrm{t}, 4 \mathrm{H},{ }^{3} J_{\mathrm{HH}}\right.$ $=8.0 \mathrm{~Hz}), 3.62\left(\mathrm{dd}, 2 \mathrm{H},{ }^{3} J_{\mathrm{HH}}=12.4 \mathrm{~Hz},{ }^{3} J_{\mathrm{HH}}=6.0 \mathrm{~Hz}\right)$, $4.02\left(\mathrm{t}, 2 \mathrm{H},{ }^{3} J_{\mathrm{HH}}=11.6 \mathrm{~Hz}\right), 4.41(\mathrm{~m}, 2 \mathrm{H}), 5.36(\mathrm{t}, 2 \mathrm{H}$, $\left.{ }^{3} J_{\mathrm{HH}}=6.4 \mathrm{~Hz}\right), 5.66(\mathrm{~s}, 2 \mathrm{H}), 6.12(\mathrm{~s}, 2 \mathrm{H}), 7.06\left(\mathrm{~d}, 4 \mathrm{H},{ }^{3} J_{\mathrm{HH}}\right.$ $=7.2 \mathrm{~Hz}, o-\mathrm{Ph} H), 7.33\left(\mathrm{t}, 2 \mathrm{H},{ }^{3} J_{\mathrm{HH}}=7.2 \mathrm{~Hz}, p-\mathrm{Ph} H\right), 7.39$ $\left(\mathrm{t}, 4 \mathrm{H},{ }^{3} J_{\mathrm{HH}}=7.2 \mathrm{~Hz}, m-\mathrm{Ph} H\right) ;{ }^{13} \mathrm{C}\left\{{ }^{1} \mathrm{H}\right\} \mathrm{NMR}\left(\mathrm{CDCl}_{3}, 100\right.$ $\mathrm{MHz}): \delta-4.8\left(\mathrm{SiCH}_{3}\right),-4.5\left(\mathrm{SiCH}_{3}\right), 18.1\left(\mathrm{SiC}\left(\mathrm{CH}_{3}\right)_{3}\right)$,

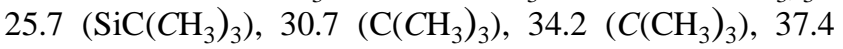
$\left(\mathrm{CH}_{2}\right.$ in pyrroloimidazolone ring), $67.1\left(\mathrm{~N}-\mathrm{CH}_{2}\right), 68.2(\mathrm{CH}$ in pyrroloimidazolone ring), $69.3 \quad(\mathrm{CH}$ in pyrroloimidazolone ring), $95.6(\mathrm{~N}-\mathrm{CH}-\mathrm{N}), 121.9(p-\mathrm{PhC})$, $127.5(\mathrm{o}-\mathrm{PhC}), 128.3(\mathrm{ArC}), 129.4(\mathrm{~m}-\mathrm{PhC}), 134.0$ (ipso$\mathrm{PhC}$ ), 144.2 ( $\mathrm{ArC}$ attached to pyrroloimidazolone ring), 146.0 (ArC attached to $t$ - $\mathrm{Bu}$ ), 152.0 ( $\mathrm{ArC}$ attached to $\mathrm{Pd}$ ), $169.6(C=\mathrm{O})$. Anal. Calcd for $\mathrm{C}_{46} \mathrm{H}_{65} \mathrm{ClN}_{4} \mathrm{O}_{4} \mathrm{PdSi}_{2}$ : C, 59.02; H, 7.00; N, 5.99. Found: C, 59.04; H, 7.07; N, 5.97; Following the procedure described for the preparation of 1-OTf, reaction of the chloride complex (187 mg, 0.200 mmol) with silver trifluoromethanesulfonate $(62.4 \mathrm{mg}$, $0.243 \mathrm{mmol})$ in a mixture of $\mathrm{CH}_{2} \mathrm{Cl}_{2}(19.8 \mathrm{~mL})$ and water $(0.2 \mathrm{~mL})$ at room temperature for $24 \mathrm{~h}$ gave 4-OTf $(206 \mathrm{mg}$, $97 \%$ ) as a white powder after trituration of the crude product with ether and hexane. Mp: 201-203 ${ }^{\circ} \mathrm{C}$ (dec.). MS (FAB): $m / z 899\left([\mathrm{M}-\mathrm{OTf}]^{+}\right) . \quad[\alpha]_{\mathrm{D}}{ }^{25}-97(c \quad 0.52$, $\left.\mathrm{CH}_{2} \mathrm{Cl}_{2}\right) .{ }^{1} \mathrm{H} \mathrm{NMR}\left(\mathrm{CDCl}_{3}, 500 \mathrm{MHz}\right): \delta 0.12(\mathrm{~s}, 12 \mathrm{H}$, $\left.\mathrm{SiCH}_{3}\right), 0.73\left(\mathrm{~s}, 9 \mathrm{H}, \mathrm{C}\left(\mathrm{CH}_{3}\right)_{3}\right), 0.89\left(\mathrm{~s}, 18 \mathrm{H}, \mathrm{SiC}\left(\mathrm{CH}_{3}\right)_{3}\right)$, $2.55(\mathrm{~m}, 2 \mathrm{H}), 2.87\left(\mathrm{dd}, 2 \mathrm{H},{ }^{3} J_{\mathrm{HH}}=13.5 \mathrm{~Hz},{ }^{3} J_{\mathrm{HH}}=8.2 \mathrm{~Hz}\right)$, $3.56\left(\mathrm{dd}, 2 \mathrm{H},{ }^{3} J_{\mathrm{HH}}=13.5 \mathrm{~Hz},{ }^{3} J_{\mathrm{HH}}=7.7 \mathrm{~Hz}\right), 4.05(\mathrm{dd}, 4 \mathrm{H}$, $\left.{ }^{3} J_{\mathrm{HH}}=13.5 \mathrm{~Hz},{ }^{3} J_{\mathrm{HH}}=3.2 \mathrm{~Hz}\right), 4.91\left(\mathrm{~d}, 2 \mathrm{H},{ }^{3} J_{\mathrm{HH}}=9.0 \mathrm{~Hz}\right)$, 6.26 (s, 2H), 6.29 (br s, 2H), 7.29 (br m, 2H, p- $\mathrm{PhH}$ ), 7.43 (br m, 8H,o- and $m-\mathrm{Ph} H) ;{ }^{13} \mathrm{C}\left\{{ }^{1} \mathrm{H}\right\}$ NMR $\left(\mathrm{CDCl}_{3}, 125\right.$ $\mathrm{MHz}): \delta-4.7\left(\mathrm{SiCH}_{3}\right),-4.6\left(\mathrm{SiCH}_{3}\right), 18.4\left(\mathrm{SiC}\left(\mathrm{CH}_{3}\right)_{3}\right)$,

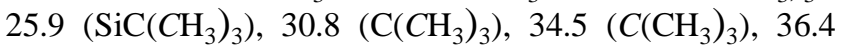
$\left(\mathrm{CH}_{2}\right.$ in pyrroloimidazolone ring), $67.2\left(\mathrm{~N}-\mathrm{CH}_{2}\right), 70.1(\mathrm{CH}$ in pyrroloimidazolone ring), $70.5 \quad(\mathrm{CH}$ in pyrroloimidazolone ring), $90.2(\mathrm{~N}-\mathrm{CH}-\mathrm{N}), 120.4\left(\mathrm{q},{ }^{1} J_{\mathrm{CF}}=\right.$ $\left.319 \mathrm{~Hz}, \mathrm{O}_{3} \mathrm{SCF}_{3}\right), 121.2(p-\mathrm{PhC}), 123.6(\mathrm{ArC}), 127.2(o-$ $\mathrm{Ph} C), 129.4(\mathrm{~m}-\mathrm{Ph} C), 135.2$ (ipso-PhC), 141.2 ( $\mathrm{ArC}$ attached to $\mathrm{Pd}$ ), 142.1 (ArC attached to pyrroloimidazolone ring), 148.7 ( $\mathrm{Ar} C$ attached to $t$ - $\mathrm{Bu}), 168.5(C=\mathrm{O})$. Anal. Calcd for $\mathrm{C}_{47} \mathrm{H}_{65} \mathrm{~F}_{3} \mathrm{~N}_{4} \mathrm{O}_{7} \mathrm{PdSSi}_{2} \cdot \mathrm{H}_{2} \mathrm{O}: \mathrm{C}, 52.87 ; \mathrm{H}, 6.32 ; \mathrm{N}$, 5.25. Found: C, 53.02; H, 6.30; N, 5.31.

General Procedure for the Michael Reactions. To a solution of the catalyst ( 0.005 equiv.) in either toluene or benzene $(2.0 \mathrm{~mL})$ were added the cyano ester $(1.0$ equiv.), the Michael acceptor (1.5 equiv.), and finally Hünig's base (0.1 equiv.). The reaction mixture was stirred at $25{ }^{\circ} \mathrm{C}$ for an appropriate time. After removal of the solvent, the residue was purified by Kugelrohr distillation to give the desired product.

Ethyl 2-cyano-2-methyl-5-oxohexanoate (10a). Colorless oil. $[\alpha]_{\mathrm{D}}{ }^{25}-3.71\left(c 1.04, \mathrm{CHCl}_{3}\right) .{ }^{1} \mathrm{H}$ NMR $\left(\mathrm{CDCl}_{3}, 400 \mathrm{MHz}\right): \delta 1.34\left(\mathrm{t}, 3 \mathrm{H},{ }^{3} J_{\mathrm{HH}}=7.2 \mathrm{~Hz}, \mathrm{CH}_{3}\right)$, $1.61\left(\mathrm{~s}, 3 \mathrm{H},-\mathrm{C}(\mathrm{CN}) \mathrm{CH}_{3}\right), 2.02-2.28\left(\mathrm{~m}, 2 \mathrm{H},-\mathrm{CH}_{2}-\right), 2.19$ $\left(\mathrm{s}, 3 \mathrm{H},-\mathrm{C}(\mathrm{O}) \mathrm{CH}_{3}\right), 2.56-2.75\left(\mathrm{~m}, 2 \mathrm{H},-\mathrm{CH}_{2}-\right), 4.27$ (q, 2H, 
$\left.{ }^{3} J_{\mathrm{HH}}=7.2 \mathrm{~Hz},-\mathrm{OCH}_{2} \mathrm{CH}_{3}\right) ;{ }^{13} \mathrm{C}\left\{{ }^{1} \mathrm{H}\right\} \mathrm{NMR}\left(\mathrm{CDCl}_{3}, 100\right.$ $\mathrm{MHz}): \delta 14.0,23.5,30.0,31.5,39.1,43.0,62.9,119.4$, 168.7, 205.6. The enantiomeric excess was determined by GC analysis using a chiral stationary phase column (Cyclodex CB) to be $81 \%$ ee. The absolute configuration was assigned on the basis of the optical rotation. ${ }^{4}$

Isopropyl 2-cyano-2-methyl-5-oxohexanoate (10b). Colorless oil. $[\alpha]_{\mathrm{D}}{ }^{25}-4.07\left(c\right.$ 1.03, $\left.\mathrm{CHCl}_{3}\right) .{ }^{1} \mathrm{H}$ NMR $\left(\mathrm{CDCl}_{3}, 500 \mathrm{MHz}\right): \delta 1.31\left(\mathrm{~d}, 3 \mathrm{H},{ }^{3} J_{\mathrm{HH}}=6.0 \mathrm{~Hz}\right.$, $\left.-\mathrm{OCH}\left(\mathrm{CH}_{3}\right)_{2}\right), 1.32\left(\mathrm{~d}, 3 \mathrm{H},{ }^{3} \mathrm{~J}_{\mathrm{HH}}=6.0 \mathrm{~Hz},-\mathrm{OCH}\left(\mathrm{CH}_{3}\right)_{2}\right)$, $1.56\left(\mathrm{~s}, 3 \mathrm{H},-\mathrm{C}(\mathrm{CN}) \mathrm{CH}_{3}\right), 2.02-2.25\left(\mathrm{~m}, 2 \mathrm{H},-\mathrm{CH}_{2}-\right), 2.19$ (s, $\left.3 \mathrm{H},-\mathrm{C}(\mathrm{O}) \mathrm{CH}_{3}\right), 2.56-2.73\left(\mathrm{~m}, 2 \mathrm{H},-\mathrm{CH}_{2}-\right.$ ), 5.07 (sept, $\left.1 \mathrm{H},{ }^{3} J_{\mathrm{HH}}=6.0 \mathrm{~Hz},-\mathrm{OCH}\left(\mathrm{CH}_{3}\right)_{2}\right) ;{ }^{13} \mathrm{C}\left\{{ }^{1} \mathrm{H}\right\} \mathrm{NMR}\left(\mathrm{CDCl}_{3}\right.$, $125 \mathrm{MHz}): \delta 21.6,21.7,23.6,30.1,31.5,39.2,43.3,70.9$, $119.3,167.9,205.3$. The enantiomeric excess was determined by GC analysis using a chiral stationary phase column (Cyclodex CB) to be $80 \%$ ee. The absolute configuration was assigned on the basis of the optical rotation. ${ }^{4}$

Diisopropylmethyl 2-cyano-2-methyl-5-oxohexanoate (10c). Colorless oil. $[\alpha]_{\mathrm{D}}{ }^{26}-0.43(c$ 1.05, $\left.\mathrm{CHCl}_{3}\right) .{ }^{1} \mathrm{H} \mathrm{NMR}\left(\mathrm{CDCl}_{3}, 500 \mathrm{MHz}\right): \delta 0.83-0.88(\mathrm{~m}$, $\left.12 \mathrm{H},-\mathrm{OCH}\left(\mathrm{CH}\left(\mathrm{CH}_{3}\right)_{2}\right)_{2}\right), \quad 1.56$ (s, $\left.3 \mathrm{H},-\mathrm{C}(\mathrm{CN}) \mathrm{CH}_{3}\right)$, 1.91-1.95 (m, 2H, $\left.-\mathrm{OCH}\left(\mathrm{CH}\left(\mathrm{CH}_{3}\right)_{2}\right)_{2}\right), 1.96-2.20(\mathrm{~m}, 2 \mathrm{H}$, $\left.-\mathrm{CH}_{2}-\right), 2.12\left(\mathrm{~s}, 3 \mathrm{H},-\mathrm{C}(\mathrm{O}) \mathrm{CH}_{3}\right), 2.52-2.67(\mathrm{~m}, 2 \mathrm{H}$, $\left.-\mathrm{CH}_{2}-\right), 4.59\left(\mathrm{t}, 1 \mathrm{H},{ }^{3} J_{\mathrm{HH}}=6.0 \mathrm{~Hz},-\mathrm{OCH}\left(\mathrm{CH}\left(\mathrm{CH}_{3}\right)_{2}\right)_{2}\right)$; ${ }^{13} \mathrm{C}\left\{{ }^{1} \mathrm{H}\right\}$ NMR (CDCl $\left.3,125 \mathrm{MHz}\right): \delta$ 17.1. 17.2, 19.6, 19.7, 24.0, 29.4, 29.5, 30.0, 31.3, 39.3, 43.4, 86.0, 119.3, 168.5, 205.2. The enantiomeric excess was determined by GC analysis using a chiral stationary phase column (Cyclodex CB) to be $82 \%$ ee. The absolute configuration was assigned on the basis of the optical rotation. ${ }^{4}$

Isopropyl 2-cyano-2-methyl-5-oxoheptanoate (12). Colorless oil. $[\alpha]_{\mathrm{D}}{ }^{26}-4.13\left(c 1.06, \mathrm{CHCl}_{3}\right) .{ }^{1} \mathrm{H}$ NMR $\left(\mathrm{CDCl}_{3}, 500 \mathrm{MHz}\right): \delta 1.07\left(\mathrm{t}, 3 \mathrm{H},{ }^{3} J_{\mathrm{HH}}=7.3 \mathrm{~Hz}\right.$, $\left.-\mathrm{C}(\mathrm{O}) \mathrm{CH}_{2} \mathrm{CH}_{3}\right), 1.31\left(\mathrm{~d}, 3 \mathrm{H},{ }^{3} \mathrm{~J}_{\mathrm{HH}}=6.0 \mathrm{~Hz},-\mathrm{OCH}\left(\mathrm{CH}_{3}\right)_{2}\right)$, $1.32\left(\mathrm{~d}, 3 \mathrm{H},{ }^{3} \mathrm{~J}_{\mathrm{HH}}=6.0 \mathrm{~Hz},-\mathrm{OCH}\left(\mathrm{CH}_{3}\right)_{2}\right), 1.60(\mathrm{~s}, 3 \mathrm{H}$, $\left.-\mathrm{C}(\mathrm{CN}) \mathrm{CH}_{3}\right), 2.03-2.26\left(\mathrm{~m}, 2 \mathrm{H},-\mathrm{CH}_{2}-\right), 2.47\left(\mathrm{q}, 3 \mathrm{H},{ }^{3} J_{\mathrm{HH}}\right.$ $\left.=7.3 \mathrm{~Hz},-\mathrm{C}(\mathrm{O}) \mathrm{CH}_{2} \mathrm{CH}_{3}\right), 2.52-2.67\left(\mathrm{~m}, 2 \mathrm{H},-\mathrm{CH}_{2}-\right), 5.07$ (sept, $\left.1 \mathrm{H},{ }^{3} \mathrm{~J}_{\mathrm{HH}}=6.0 \mathrm{~Hz},-\mathrm{OCH}\left(\mathrm{CH}_{3}\right)_{2}\right) ;{ }^{13} \mathrm{C}\left\{{ }^{1} \mathrm{H}\right\} \mathrm{NMR}$ $\left(\mathrm{CDCl}_{3}, 125 \mathrm{MHz}\right): \delta 7.87,21.5,21.6,23.5,31.6,36.1$, 37.9, 43.3, 70.9, 119.3, 167.9, 208.0. The enantiomeric excess was determined by GC analysis using a chiral stationary phase column (Cyclodex CB) to be $83 \%$ ee. The absolute configuration was assigned on the basis of the optical rotation. ${ }^{4}$

$\mathrm{X}$-ray structure determination. A single crystal suitable for X-ray structure analysis was obtained by cooling a hot solution of $\mathbf{1 - C l}$ in toluene. The data were collected on a Rigaku Mercury CCD area detector with graphite monochromated Mo-K $\alpha$ radiation $(\lambda=0.71070 \AA)$ at a temperature of $173 \mathrm{~K}$. The structure was solved by direct methods (SIR97 $)$ and expanded using Fourier techniques (DIRDIF99). The non-hydrogen atoms were refined anisotropically and hydrogen atoms were refined using the riding model. Neutral atom scattering factors were taken from Cromer and Waber. ${ }^{6}$ Anomalous dispersion effects were included in Fcalc. $^{7}$ All calculations were performed using the CrystalStructure ${ }^{8}$ crystallographic software package. Crystal data of 1Cl•toluene: $\mathrm{C}_{41} \mathrm{H}_{45} \mathrm{ClN}_{4} \mathrm{O}_{2} \mathrm{Pd}$, orthorhombic, $P 2_{1} 2_{1} 2_{1}$ (No. 19), $a=12.2613$ (5) $\AA, b=12.4185$ (5) $\AA, c=23.9133$ (11) $\AA, V=3641.2(3) \AA^{3}, Z=4, R 1=0.023, w R 2=0.060$, $\mathrm{GOF}=0.94$.

\section{Reference}

1 Lindoy, L. F.; Meehan, G. V.; Svenstrup, N. Synthesis 1998, 1029.

2 Ukai, T.; Kawazura, H. Ishii, Y. J. Organomet. Chem. 1974, 65, 253.

3 (a) Uozumi, Y.; Mizutani, K.; Nagai, S. Tetrahedron Lett. 2001, 42, 407; (b) Uozumi, Y.; Yasoshima, K.; Miyachi, T.; Nagai, S. Tetrahedron Lett. 2001, 42, 411.

4 (a) Sawamura, M.; Hamashima, H.; Ito, Y. J. Am. Chem. Soc. 1992, 114, 8295; (b) Sawamura, M.; Hamashima, H.; Ito, Y. Tetrahedron 1994, 50, 4439

5 Altomare, A.; Burla, M.; Camalli, M.; Cascarano, G.; Giacovazzo, C.; Guagliardi, A.; Moliterni, A.; Polidori, G.; Spagna, R. J. Appl. Cryst. 1999, 32, 115.

6 Cromer, D. T.; Waber, J. T. International Tables for $X$-ray Crystallography, Kynoch Press, Birmingham (UK), 1974, Vol. 4.

7 Ibers, J. A.; Hamilton, W. C. Acta Crystallogr. 1964, $17,781$.

8 Crystal Structure Analysis Package, Rigaku and Rigaku/MSC (2000-2002). 